\section{Build your own B cell}

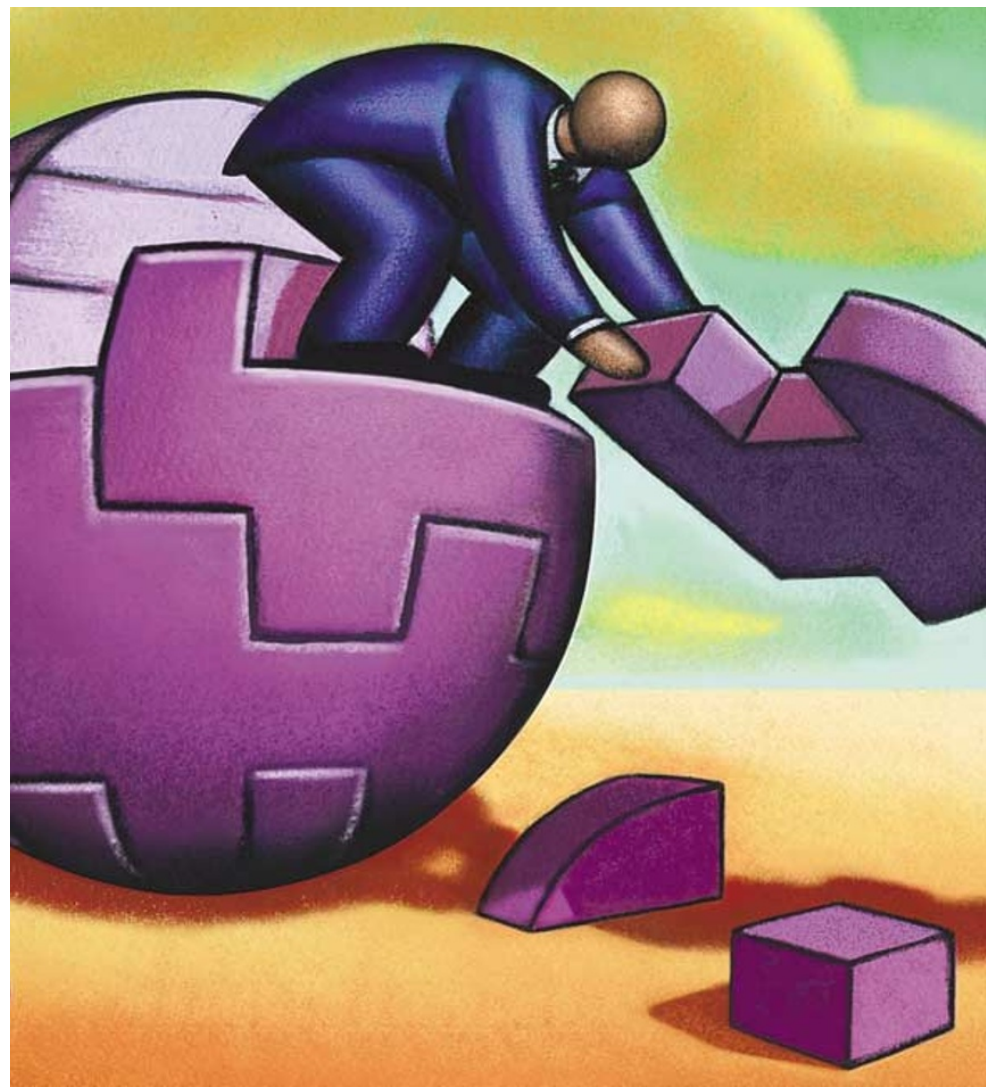

Our map of antigen-receptor signalling pathways is based largely on studies in which the function of signalling molecules was disrupted. This knockout approach allows the ordering of components in a pathway, but it can tell us little about feedback mechanisms that might exist. This led Rolli and colleagues at the University of Freiburg to devise an alternative approach - the reconstruction of B-cell receptor (BCR) signalling pathways in Drosophila cells. The new system has produced some surprising results, which are reported in Molecular Cell.

Interference from endogenous signalling pathways is a potential problem when expressing receptors and signalling components in another vertebrate cell type. To minimize this, Rolli et al. used Drosophila Schneider S2 cells. These cells are used commonly for large-scale protein production, so it was probable that they would be able to 'cope' with the cotransfection of several foreign genes. Expression vectors containing a copper-sulphate-inducible promoter were used to express the BCR and various signalling components in S2 cells.

According to present models, BCR signal transduction is initiated by phosphorylation of the two tyrosine residues in the ITAMs (immunoreceptor tyrosine-based activation motifs) of $\operatorname{Ig} \alpha / \beta$ (the paired signalling subunits of the BCR) by the membrane-associated SRC-family protein tyrosine kinase (PTK) LYN. This is thought to allow recruitment of the cytosolic PTK SYK and activation of downstream effector enzymes. But, by transfecting S2 cells with the BCR and various combinations of wildtype or mutated forms of $\operatorname{Ig} \alpha / \beta$, LYN and SYK, the authors found that LYN tends to phosphorylate only the first tyrosine of the ITAM, whereas SYK can phosphorylate both tyrosines. Therefore, SYK can phosphorylate the BCR independent of LYN, and it is a more probable candidate for the first step in BCR signal transduction.

Further experiments showed that the SRC-homology 2 (SH2) domains of SYK, which bind to phosphorylated ITAMs, are required to optimize the activity of its catalytic domain. This implies that SYK can regulate its own activity, because its product — phosphorylated ITAMs - induces the further activation and recruitment of SYK. This positivefeedback loop has the potential to
HIV

\section{Immunological burn-out}

The failure to control HIV-1 infection results in immunodeficiency and destruction of the immune system in infected individuals. In addition to infecting and killing $\mathrm{CD}^{+} \mathrm{T}$ cells directly, HIV-1 causes chronic immune activation, which has been proposed to contribute to the immunodeficiency. Now, Rene van Lier's group have shown that persistent immune activation - in this study induced by chronic co-stimulation through CD27-CD70 interactions - can indeed result in lethal immunodeficiency. Members of the tumour-necrosis factor receptor family, which includes CD27, are involved in the regulation of diverse immunological processes, including immune-cell proliferation and survival. CD70, the ligand for CD27, is expressed by activated lymphocytes after antigenic stimulation. Here, the authors used $C d 70$ transgenic mice, in which $B$ cells chronically express CD70, as a model to assess the effects of persistent immune activation on the immune system.

$C d 70$-transgenic mice had increased numbers of effector $\mathrm{T}$ cells in their spleens and peripheral lymph nodes, owing to increased T-cell proliferation. With time, the number of naive $\mathrm{T}$ cells in the secondary lymphoid organs decreased, as did naive T-cell production, but effector-memory $\mathrm{T}$ cells accumulated. Further experiments showed that this excessive production of effector-memory $T$ cells was dependent on CD27-CD70 interactions and on the presence of foreign antigens, but was independent of interferon- $\gamma$.
How do these changes in T-cell populations affect the health of the $C d 70$ transgenic mice? By 20 weeks of age, most of the mice suffered from Pneumocystis carinii pneumonia - an opportunistic infection that is commonly seen in situations of severe T-cell immunodeficiency - which resulted in their premature death at around 28 weeks of age.

This study shows that persistent immune activation, as has been proposed to occur during chronic HIV-1 infection, is sufficient to cause lethal immunodeficiency. As activated T cells from HIV-1-infected individuals have been shown to express increased levels of CD70, the authors suggest that CD27-CD70 interactions could be targeted to avoid the negative effects of persistent immune activation.

Jenny Buckland

(2) References and links

ORIGINAL RESEARCH PAPER Tesselaar, K. et al. Lethal T-cell immunodeficiency induced by chronic costimulation via CD27-CD70 interactions. Nature Immunol. 9 December 2002 (DOI: 10.1038/ni869) 
amplify BCR signal transduction and might have a role in the initiation of BCR signals.

With such a powerful positivefeedback mechanism in place, BCR signalling could easily get out of control. Experiments in $\mathrm{S} 2$ cells indicated that SHP1 (SH2-domain-containing protein tyrosine phosphatase 1) might be the crucial brake. By dephosphorylating ITAMs, SHP1 could disrupt the positive-feedback loop of SYK activation. As Michael Reth discusses in a recent review in Nature Immunology, inhibition of SHP1 by hydrogen peroxide might allow the initiation of BCR signalling.

So, the S2 'gain of function' system has provided a fresh view of early BCR signal transduction, and the authors hope that it will prove to be a useful tool for the signalling community.

Jennifer Bell

(2) References and links ORIGINAL RESEARCh PAPER Rolli, $v$. et al. Amplification of B-cell antigen-receptor signaling by a Syk/TAM positive-feedback loop. Mol. Cell 10, 1057-1069 (2002)

FURTHER READING Reth, M. Hydrogen peroxide as a second messenger in lymphoctye activation. Nature Immunol. 3, 1129-1134 (2002). WEB SITES

Michael Reth's lab:

http://www.immunbio.mpg.de/groups/reth/reth_fl ash.html

Alliance for Cellular Signaling:

http://www.signaling-gateway.org/

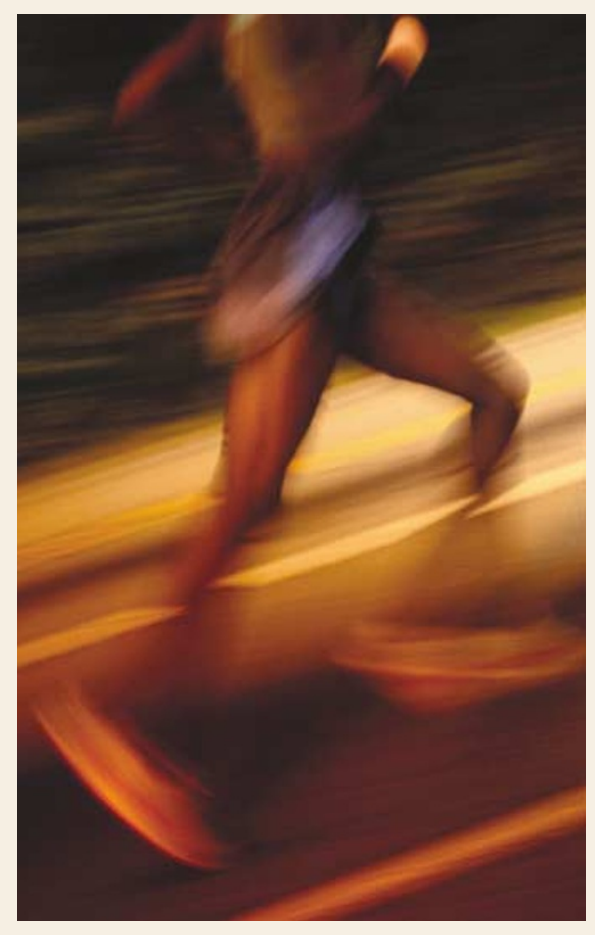

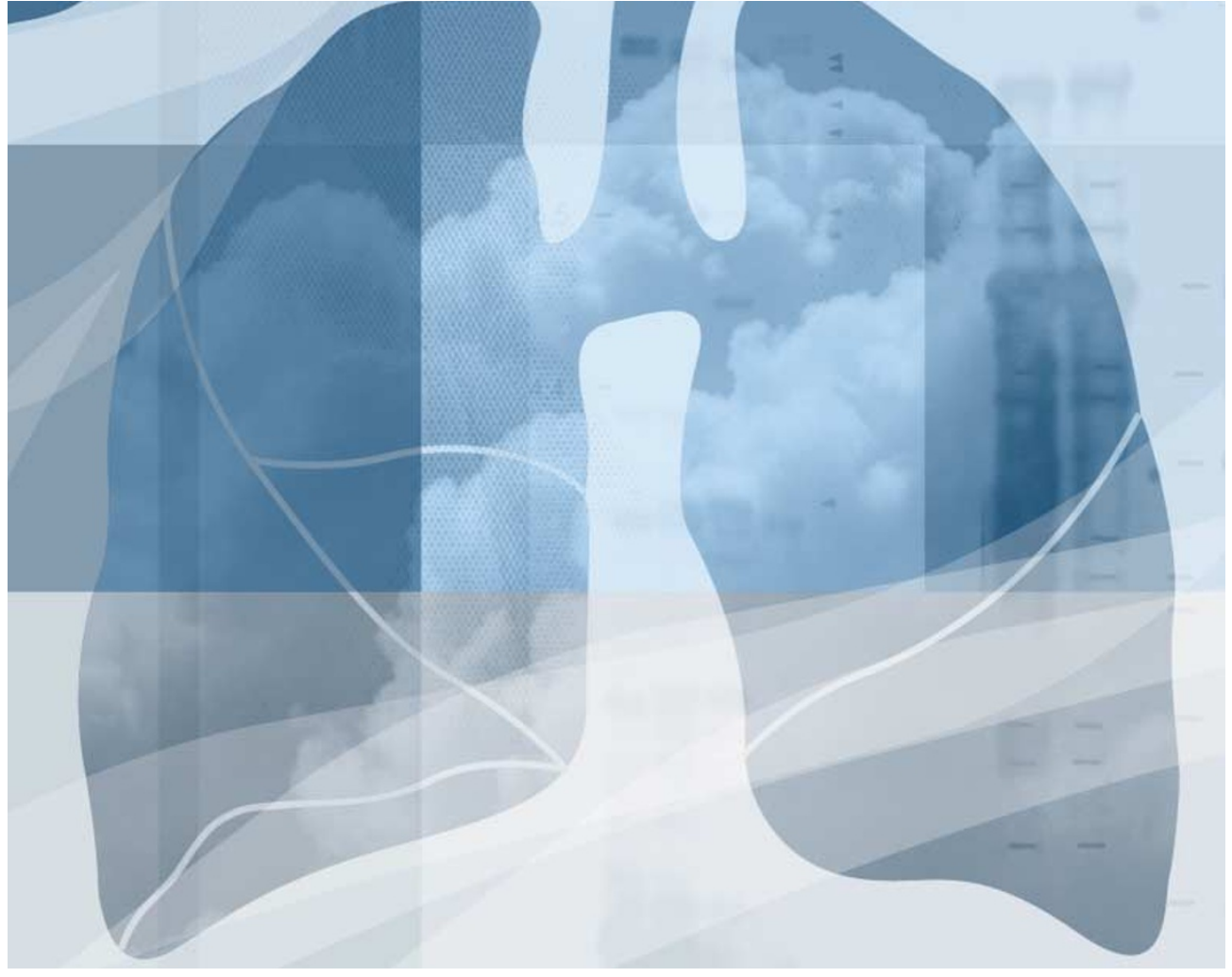

MUCOSAL IMMUNOLOGY

\section{Preferential pulmonary priming}

In studies that might be relevant to human

$T$ helper $2\left(T_{H} 2\right)$-dominated immune responses of the lungs, such as allergic asthma, Constant et al. report in the Journal of Clinical Investigation that not only do lung antigen-presenting cells (APCs) preferentially prime $T_{H} 2$, rather than $T_{H} 1$, responses, but that they do so in situ, without migrating to draining lymph nodes. This feature of the pulmonary tract might hold true for other mucosal surfaces, such as the digestive tract.

It is a common phenomenon that the intranasal delivery of most protein antigens seems to prime preferentially for $\mathrm{T}_{\mathrm{H}} 2$ responses, characterized by eosinophilia and interleukin- 5 (IL-5) production. However, Constant et al. now show that the intranasal delivery of a ' $\mathrm{T}_{\mathrm{H}} 1$ inducing' antigen (such as Leishmania major pathogens in C57BL/6 mice) also induces a $\mathrm{T}_{\mathrm{H}} 2$ response in the lungs, compared with subcutaneous delivery, which induces a response characterized by high levels of interferon- $\gamma\left(T_{H} 1\right)$. What can account for these results? Both the type of APC and the cytokine microenvironment during antigen presentation are known to affect $\mathrm{T}_{\mathrm{H}}$-cell differentiation.

Several potential APCs exist in the lungs, including alveolar macrophages, tissue monocytes, epithelial cells and dendritic cells (DCs). Using FACS analysis, Constant et al. show that the APCs that take up and present $L$. major antigens to $\mathrm{T}$ cells have a CD1 $1 \mathrm{c}^{\text {hi }} \mathrm{CD} 11 \mathrm{~b}^{\text {low }}$ phenotype, express DEC205 and F4/80, and are negative for CD8 $\alpha$ and CD14. This indicates that they have a DC-like phenotype.
How do these cells respond to antigen uptake? Surprisingly, only small changes in the level of expression of activation markers were observed after intranasal delivery of antigen, which indicates that the APCs do not undergo marked maturation/differentiation. Furthermore, in the absence of a pro-inflammatory stimulus, only a small number of the APCs migrate to draining lymph nodes, an event that is associated usually with the maturation of DCs.

However, using mice that are deficient for lymphotoxin- $\alpha$ (which lack lymph nodes) and that have been splenectomized, the authors show that migration of APCs to secondary lymphoid tissues is not necessary for pulmonary T-cell priming, in contrast to priming after subcutaneous or intraperitoneal delivery of antigen.

Finally, the authors investigated the cytokines that are produced by these APCs in situ. There is a marked upregulation of expression of messenger RNA encoding IL-6 and IL-10 after antigen uptake; both of these cytokines are known to be involved in promoting $\mathrm{T}_{\mathrm{H}}$ 2-cell differentiation.

So, in the absence of pro-inflammatory stimuli, lung CD11 $\mathrm{c}^{\text {hi }} \mathrm{CD} 11 \mathrm{~b}^{\text {low }} \mathrm{APCs}$ present antigen to $T$ cells in situ and have an inherent bias to induce $\mathrm{T}_{\mathrm{H}} 2$ cells through the production of IL- 6 and IL-10.

\section{(2) References and links}

Kirsty Minton

ORIGiNAL RESEARCH PAPER Constant, S. L. et al. Resident lung antigen-presenting cells have the capacity to promote $\mathrm{T}_{\mathrm{H}} 2 \mathrm{~T}$-cell differentiation in situ. J. Clin. Invest. 110, 1441-1448 (2002) FURTHER READING Moore, B. B., Moore, T. A. \& Toews, G. B. Role of T- and B-lymphocytes in pulmonary host defences. Eur. Resp. J. 18, 846-856 (2001) 